\title{
The identification of 93 day periodic photometric variability for YSO YLW 16A
}

\author{
P. Plavchan ${ }^{1}$, T. Güth ${ }^{1}$, N. Laohakunakorn ${ }^{2}$, and J. R. Parks ${ }^{3}$ \\ 1 Infrared Processing and Analysis Center, California Institute of Technology, M/C 100-22, $770 \mathrm{~S}$ Wilson Avenue, Pasadena, \\ CA 91125, USA \\ e-mail: plavchan@gmail.com \\ 2 Trinity College, Cambridge, CB2 1TQ, UK \\ 3 Georgia State University, 33 Gilmer St., Atlanta, GA 30303, USA
}

Received 16 November 2012 / Accepted 4 April 2013

\begin{abstract}
Aims. Periodic variability in young stellar objects (YSOs) offers indirect evidence for an active dynamical mechanism. Starspots, accretion, stellar companions, and disk veiling can contribute to the photometric variability of YSOs.

Methods. As part of an ongoing study of the $\rho$ Oph star forming region, we report the discovery of 92.6 day periodic variations for the Class I YSO YLW 16A, observed over a period of three years. A SED model was fit to available photometric data for the object. Results. We propose a triple-system with an inner binary with a period of 93 days eclipsed by a warped circum-binary disk. The nature of the secondary is unconstrained and could be stellar or sub-stellar. We confirm the discovery of a tertiary companion at a projected separation of $\sim 40 \mathrm{AU}$ that could account for the circum-binary disk warp. This light curve and model is similar to the model we proposed for WL 4 in previous work. Understanding these systems may lead to insights about the nature of stellar evolution and planetary formation, and provide valuable benchmarks for future theoretical modeling and near- and mid-infrared synoptic surveys of YSOs.
\end{abstract}

Key words. circumstellar matter - stars: variables: T Tauri, Herbig Ae/Be

\section{Introduction}

Star formation involves the gravitational collapse of a massive cloud core. Between this initial collapse, and the final contraction onto the main sequence, the protostar is classified as a young stellar object (YSO). These YSOs have ages of a few million years $(\sim 1-10 \mathrm{Myr})$, and are characterized by high levels of accretion, ejection, and magnetic activity, as well as photometric variability (Joy 1945). The evolution of YSOs falls broadly into four stages. Class 0 objects consist of a collapsing cloud core. Class I objects are protostars embedded inside a sphericallysymmetric infall envelope. Class II objects, also known as "classical T Tauri stars," contain a stable primordial disk. The dispersion of the stable disk reveals a Class III object, or diskless "weak-lined T Tauri star" (e.g. Adams et al. 1987). The spectral energy distribution (SED) of YSOs differ from normal stars by exhibiting an infrared excess as the circumstellar material reprocesses the central radiation. The amount of excess is strongly correlated with the evolutionary stage of the YSO. Due to the strength of the infrared emission, it is natural to study YSOs at these wavelengths.

It is widely accepted that planetary systems form out of primordial protostellar disks, and because such disks are an essential structure in the evolution of Class II YSOs, the study of YSOs can lend valuable insights into the processes by which planets form (Lin \& Papaloizou et al. 1980; Ida \& Lin 2010, and references therein). At optical wavelengths, some YSOs are observed to exhibit periodic photometric variability (e.g., Rebull 2001; Covey et al. 2006). The observed variability is generally attributed to the rotational modulation of large cold spots, hot spots, accretion and disk veiling. Photometric variability driven by rotational modulation of the proto-star are less pronounced at infrared wavelengths, thus improving sensitivity to variability driven by disk-related processes and the subject of many recent near-infrared (NIR) and Spitzer Space Telescope studies such as YSOVAR (Morales-Calderon et al. 2011; Flaherty et al. 2012; Flaherty \& Muzerolle 2010; Faesi et al. 2012).

$\rho$ Ophiuchus $(\rho \mathrm{Oph})$ is a nearby $(\sim 135 \mathrm{pc})$ star-forming region containing a few hundred such YSOs from a few Solar masses down to the free-floating planet mass regime (Mamajek 2008; Marsh et al. 2010). In this paper we investigate the YSO in $\rho$ Oph: YLW 16A. YLW 16A (= IRAS 16244-2432, 2MASS J16272802-2439335, ISO-Oph 143, IRS 44) is classified as a Class I protostar (e.g. Luhman \& Rieke 1999; Barsony et al. 2005) which has been a notable subject of a previous study at X-ray wavelengths (Grosso 2001; Imanishi et al. 2001). Imanishi et al. (2001) detected an unusual bright X-ray flare, with a peak luminosity of $1.3 \times 10^{31} \mathrm{ergs} \mathrm{s}^{-1}$. A $6.4 \mathrm{keV}$ emission line was identified, which was attributed to fluorescence of cold neutral iron in the circumstellar gas. An extended 3400 AU (Beckford et al. 2008) nebulosity has been observed around YLW 16A in the infrared ( $H$ and $K_{\mathrm{s}}$ bands; Simon et al. 1987; Lucas \& Roche 1998) and at thermal radio wavelengths (Leous et al. 1991; Girart et al. 2004). High-resolution HST NICMOS imagery, obtained June 1998, reveals two nonpoint sources separated by $0.5^{\prime \prime}$, with flux ratios of 1.5 at $1.1 \mu \mathrm{m}$ and 1.1 at $1.6 \mu \mathrm{m}$ (Allen et al. 2002). Beckford et al. (2008) interpret the second source as being due to a reflection from a dusty jet inside a bipolar cavity. However, their conclusions do not rule out the possibility of a binary companion. Herczeg et al. (2011) also find a resolved binary with CRIRES/VLT, finding the west component 
to be $0.69 \pm 0.12 \mathrm{mag}$ fainter at $M$-band, with $\mathrm{CO}$ and extended $\mathrm{H}_{2}$ emission, but no $\mathrm{CO}$ emission from the east component. Simon et al. (1987) reported a variability of $\sim 1.0 \mathrm{mag}$ in the $K_{\mathrm{s}}$ band, over a timescale of approximately six months. Finally, Evans et al. (2009) derive an extinction of $A_{V}=9.8 \mathrm{mag}$ for YLW 16A from the Spitzer c2d survey, which would correspond to a $A_{J} \sim 2$.

Doppmann et al. (2005) presented a high signal-to-noise ratio (S/N) NIR spectrum of YLW 16A, which is essentially featureless. There is, however a sharp $\mathrm{H}_{2}$ emission line at $2.1218 \mu \mathrm{m}$, characteristic of still accreting YSOs, and weak absorption features including the $\mathrm{CO} 2-0$ bandhead at $2.3 \mu \mathrm{m}$. In general, Class I spectra tend to exhibit weaker features due to veiling and continuum emission characteristic of a protostar surrounded by a thick envelope (Greene \& Lada 2000). Covey et al. (2006) measure a local standard rest velocity of $4.41 \mathrm{~km} \mathrm{~s}^{-1}$, and do not identify evidence for a double-lined spectroscopic binary companion, down to the $\sim 1-2 \mathrm{~km} \mathrm{~s}^{-1}$ level, from the structure of the radial velocity cross-correlation function in a single epoch spectrum (Covey et al. 2013, priv. comm.).

Another YSO located in $\rho$ Oph is WL 4, a Class II object, whose periodic photometric variability was discovered by Plavchan et al. (2008a) to be $130.87 \pm 0.40$ days. The authors suggest a triple-YSO model, consisting of an inner binary and a third companion further out. A circum-binary disk, tilted with respect to the binary's orbital plane by the gravitational influence of the third companion, eclipses each member of the inner binary in turn. Periodic eclipsing of a binary by a circum-binary disk is also the preferred model of the well-studied system KH-15D (Herbst et al. 2010, and references therein), as well as the recently discovered object CHS 7797 in the Orion star-forming region (Rodriguez-Ledesma et al. 2013, 2012).

In this paper, we present the discovery of periodic NIR photometric variability for YLW 16A. Our analysis parallels much of the analysis in Plavchan et al. (2008a). We present our observations in Sect. 2, and results in Sect. 3. In Sect. 4, we discuss the implications of our observations and proposed model. This discovery demonstrates that systems like YLW 16A, WL 4, KH-15D and CHS 7797 may be common in multiple star-forming regions, constituting a new class of disk eclipsing YSOs. These systems will be valuable to study the evolution of circumstellar disks around YSOs, and potentially the formation sites for circumbinary planets (Doyle et al. 2011).

\section{Observations}

The photometry for the $J, H$, and $K_{\mathrm{s}}$ bands were obtained from the Two Micron All-Sky Survey (2MASS) Calibration Point Source Working Database (Cal-PSWDB; Skrutskie et al. 2006). Between 1997 and 2001, 2MASS imaged the entire sky in three NIR bands, $J, H$, and $K_{\mathrm{s}}$. Hourly observations of 35 different calibration fields were used to calibrate the 2MASS photometry. One such field is located in $\rho$ Oph, $8.5^{\prime}$ wide in R.A. by $60^{\prime}$ long in decl., and centered at $(\mathrm{RA}, \mathrm{Dec})=\left(246.80780^{\circ},-24.68901^{\circ}\right)$. A total of 1582 independent observations were made of this field, which contains YLW 16A, as discussed in further detail in Plavchan et al. (2008a); Parks et al. (2013).

Two NACO images of YLW 16A, taken from the European Southern Observatory (ESO) archive for the instrument, were obtained for the $K_{\mathrm{s}}$ and $L$ bands (Lenzen et al. 2003; Rousset et al. 2003), as seen in Fig. 1 . The $L$ band image was obtained on 9 April 2005, while the $K_{\mathrm{s}}$ band was obtained on 30 April 2005. The NACO images allow us to measure a flux ratio between
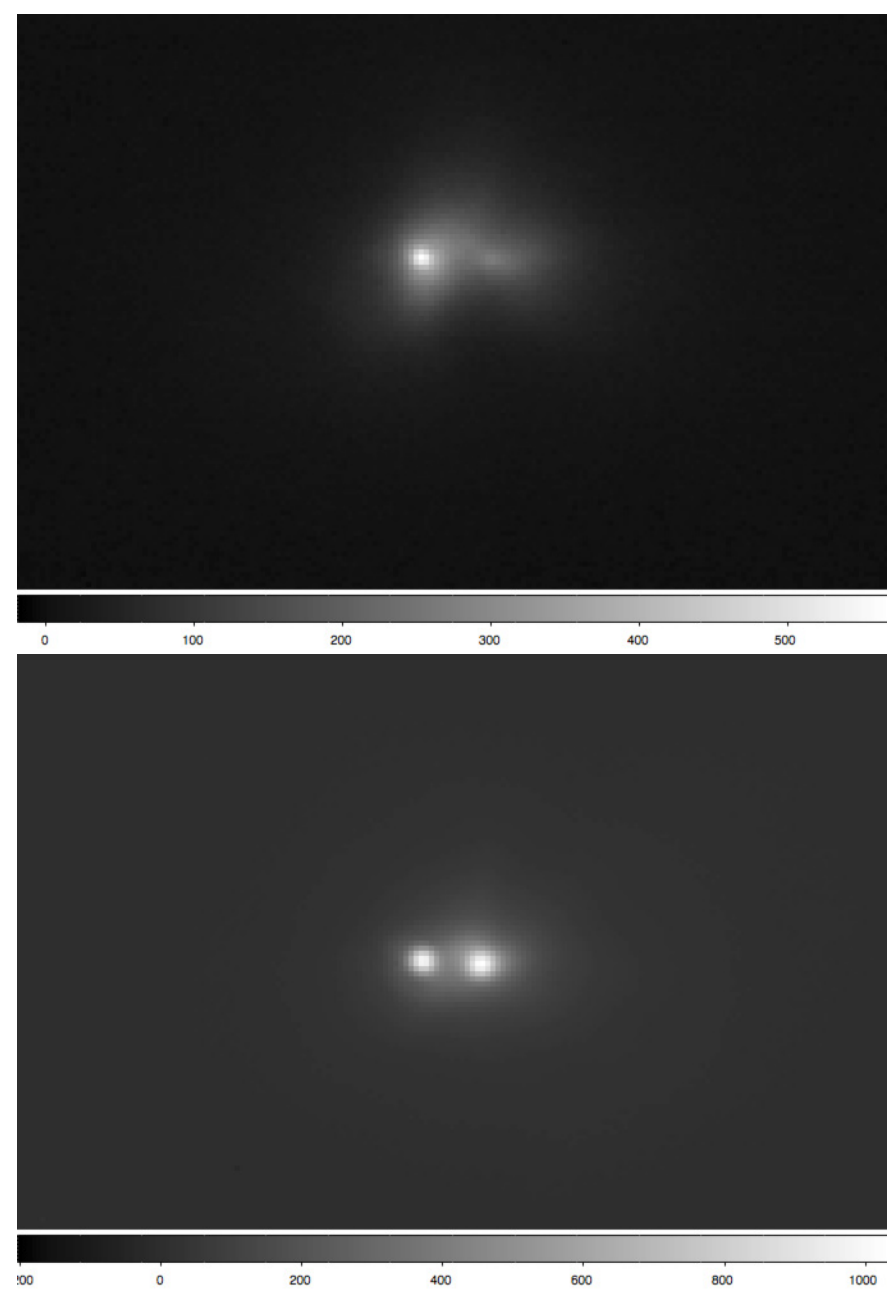

Fig. 1. NACO imagery of YLW $16 \mathrm{~A}$ in the $K_{\mathrm{s}}$ (Upper) and $L$ (Lower) bands. The separation of the two sources in the $L$ band is approximately $0.3^{\prime \prime}$ ( $\sim 0 \mathrm{AU}$ projected separation). Both these images were obtained in the faint state $(L$ band phase $=0.4656, K$ band phase $=$ 0.6920). Color bars shown correspond to non-normalized counts. North is up and east is to the left.

YLW 16AA and YLW 16AB. Using aperture photometry, we derive flux ratios from the NACO images of 0.22 and 0.98 at $K_{\mathrm{S}}$ and $L$ respectively. We calibrate to the total flux from the 2MASS $K_{\mathrm{s}}$ magnitude in the faint state (for the $K_{\mathrm{s}}$ NACO image) of $51.5 \mathrm{mJy}$ and IRAC 3.6 microns in the faint state (for $L$ ) of $695.8 \mathrm{Jy}$.

Photometry for YLW 16A was also obtained from the IRAC (3.6, 4.5, 5.8, and $8 \mu \mathrm{m})$ and MIPS $(70 \mu \mathrm{m})$ instruments on the Spitzer Space Telescope, as part of the Cores to Disks (c2d) Spitzer Space Telescope Legacy program (Evans et al. 2003; Padgett et al. 2008). YSOVAR has also obtained photometric time-series of YLW 16A at 3.6 and $4.5 \mu \mathrm{m}$ during the Spitzer Space Telescope warm mission, which will be part of a separate future publication (Morales-Calderon et al. 2011).

Finally, photometry was obtained from the literature at $10.8 \mu \mathrm{m}$ (Barsony et al. 2005), $850 \mu \mathrm{m}$ (Jorgensen et al. 2008), and $1.2 \mathrm{~mm}$ (Stanke et al. 2006). Excluding the NACO photometry, all other photometry is a blend of the YLW 16A system. The average 2MASS photometry, as well as the additional photometry values are summarized in Table 1 . 
P. Plavchan et al.: The identification of 93 day periodic photometric variability for YSO YLW 16A

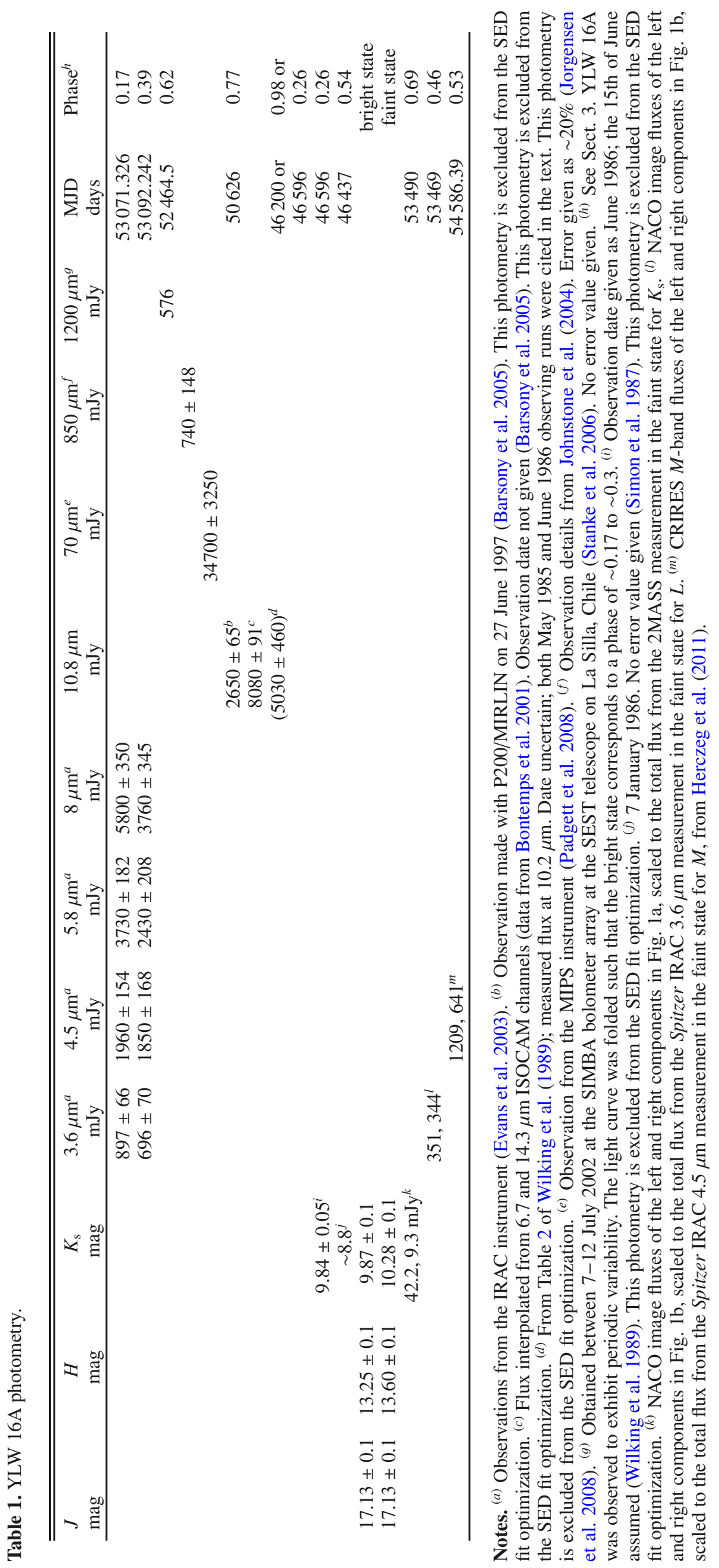


RA: 246.866684 Dec: -24.659260
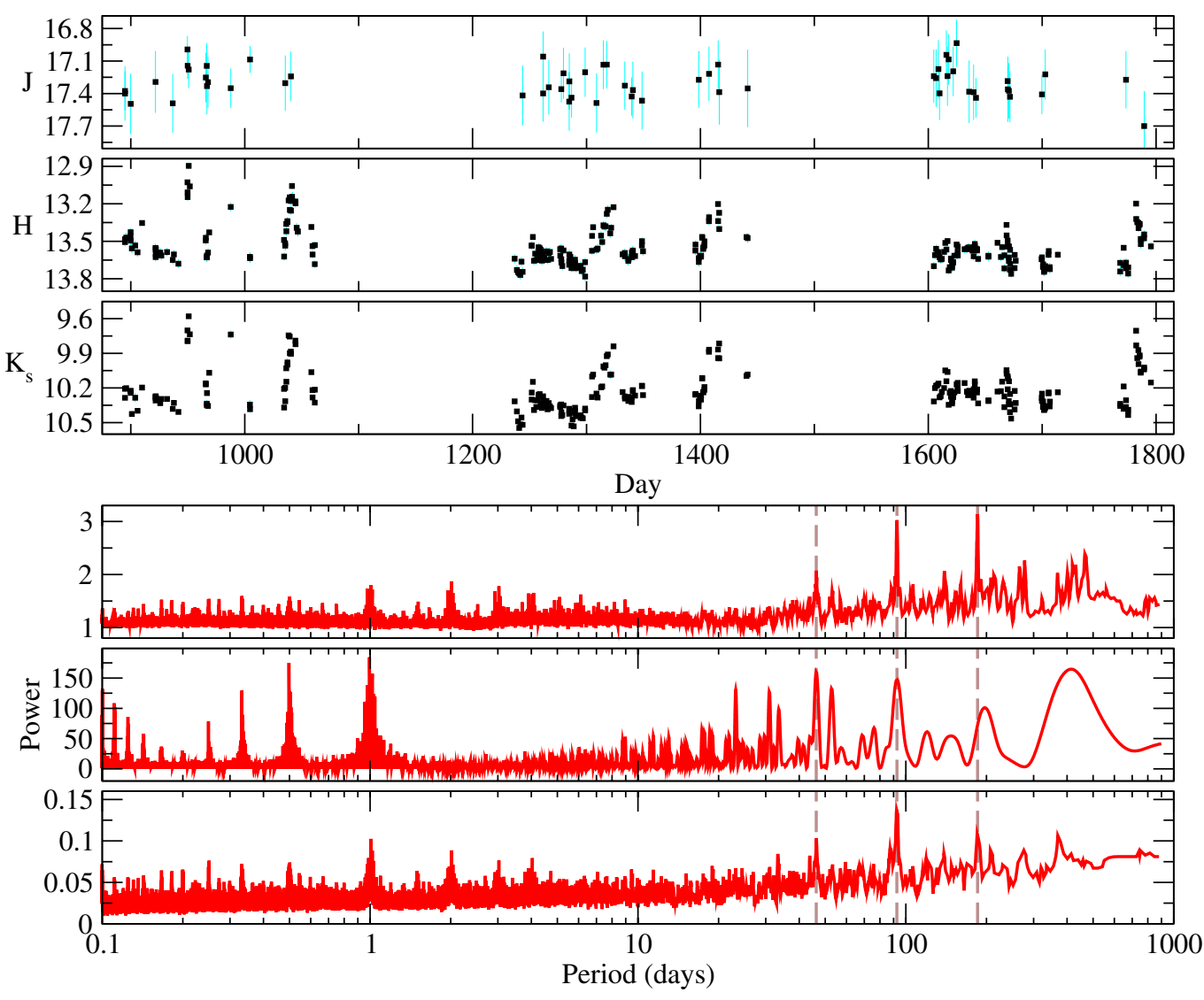

Fig. 2. Top three panels: the $J, H, \& K_{\mathrm{s}}$-band light curves for YLW 16A, generated using data from the 2MASS CalPSWDB. "Scan groups" of six measurements taken in 10 min of elapsed real time are co-added, as in Plavchan et al. (2008b). Propagated $1 \sigma$ error bars are shown in teal. Bottom three panels: Plavchan, Lomb-Scargle and Box Least Squares periodograms of the $K_{\mathrm{s}}$-band light curve (Scargle 1982; Kovacs et al. 2002; Plavchan et al. 2008b).

\section{Analysis and results}

\subsection{Periodic variability}

Periodic variability is readily apparent from a visual inspection of the YLW 16A light curve (Fig. 2). Using the LombScargle periodogram (Scargle 1982), the Box Least Squares periodogram (Kovacs et al. 2002), as well as the period-searching algorithm of Plavchan et al. (2008b), a period of $92.62 \pm$ 0.84 days is identified (also see Parks et al. 2013). Access to all three algorithms are available online in interactive form at the NASA Exoplanet Archive, and include rudimentary estimates of the false-alarm probabilities (p-values). The period and period $1 \sigma$ error are assessed from the Plavchan periodogram peak and Half-Width Half-Maximum respectively. All three algorithms detect the signal at 92.6 days, and period aliases of one-half and two times this period.

The bin-less phase dispersion minimization Plavchan periodogram is adept at detecting arbitrarily-shaped periodic signals, compared to sinusoids for Lomb-Scargle and box-like transits for BLS, and thus detects the 93-day period with higher statistical significance $(5.4 \sigma)$. All three algorithms detect aliased periods at integer fraction multiples of one day (e.g. periods of $1 / 4,1 / 3,1 / 2,2,3$, and 4 days, etc.) with statistical significance that in the case of Lomb-Scargle exceeds the 93-day signal. This is due to the long-term (e.g. 400-500 day) variations seen in the light curve aliased with the $\sim 1$ observation per day cadence. Visual inspection of the phased time-series confirms that these are aliased "false" periods. All three algorithms detect these "red-noise" long-term trends that are likely astrophysical in origin, but we do not quantify this time-scale further (Parks et al. 2013).
Figure 3 shows two repeated cycles of the phased light and color curves of YLW 16A. For JD of 2450000.0 (note, not MJD $=0$ ), the corresponding phase is 0 in Fig. 3. The average variation between bright and faint states is $\sim 0.5 \mathrm{mag}$ in the $K_{\mathrm{s}}$ band, with a maximum range of $\Delta K_{\mathrm{s}}=0.95 \mathrm{mag}$. There is also periodic variability in the $\left(H-K_{\mathrm{s}}\right)$ curve. The shape of the color variations differ from the shape of the photometric variations in the $K_{\mathrm{s}}$ band, with an approximately sinusoidal phased curve with an amplitude of $\sim 0.15 \mathrm{mag}$ and a maximum range of $\triangle\left(H-K_{\mathrm{s}}\right)=0.34 \mathrm{mag}$. The mean $K_{\mathrm{s}}$ magnitude is 10.22 and the mean $\left(J-K_{\mathrm{s}}\right)$ color is 7.07. The low $\mathrm{S} / \mathrm{N} J$ band data is due to the high extinction toward the system. There are two data points that appear to be particular errant from the phased time-series in Fig. 3 at phases of $\sim 0.7$ and 0.8 , which may indicate additional sporadic variability such as a flare that was not adequately sampled by the cadence of our observations.

The folded light and color curves show that YLW 16A has both dim and bright states similar to WL 4 as discussed in Plavchan et al. (2008a). However, the bright state of YLW 16A is about half as long in phase duration as that of WL 4, such that it appears very much like an "upside-down eclipse". Additionally, the source is redder in $H-K_{\mathrm{s}}$ when the bright state occurs, which runs counter-intuitive to models for extinction variability (Parks et al. 2013).

\subsection{SED modeling}

A model SED fit is generated, as done in Plavchan et al. (2008a). Our model SED can include up to three stellar components and five dust components, each with an independent extinction magnitude. We also allow for a variable broken power law for the 
P. Plavchan et al.: The identification of 93 day periodic photometric variability for YSO YLW 16A

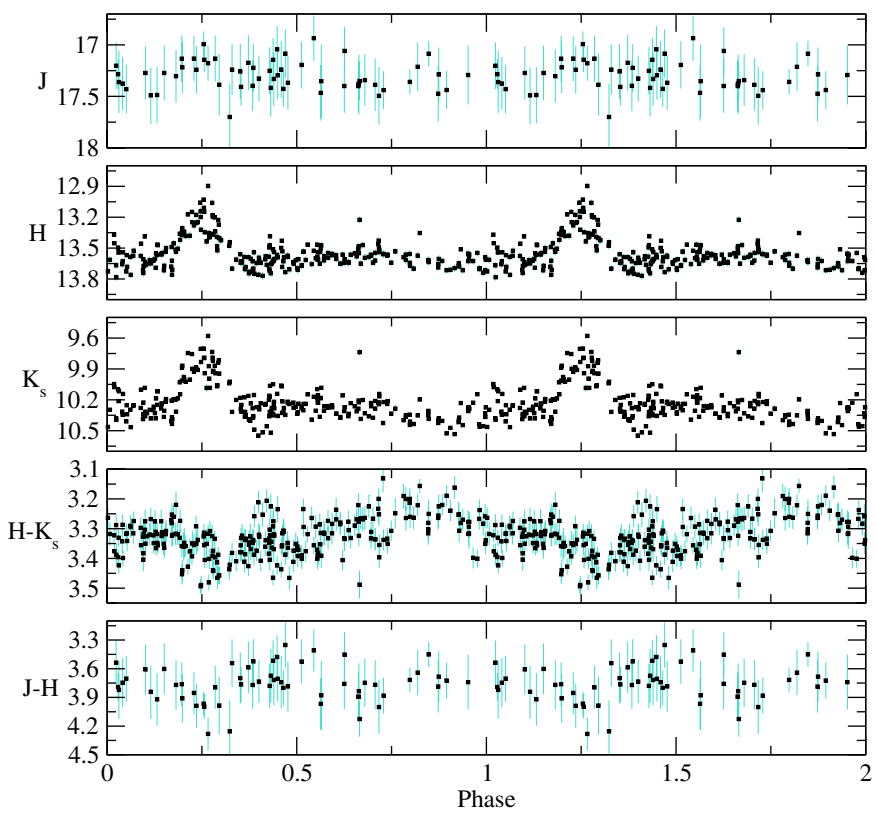

Fig. 3. From top to bottom: the $J, H, K_{\mathrm{s}}, H-K_{\mathrm{s}}, \& J-H$ phased light and color for YLW 16A, generated using data from the 2MASS CalPSWDB as in Fig. 2, folded to a period of 92.6 days and plotted as a function of phase. A second phase of the same data is repeated.

system extinction as a function of wavelength (e.g. $A_{\lambda} \propto \lambda^{\alpha_{1}, \alpha_{2}}$ for $\lambda<,\rangle \lambda_{0}$ ), and we dynamically color-correct 24 and $70 \mu \mathrm{m}$ Spitzer photometry. Reddened Phoenix NextGen (Hauschildt et al. 1999) spectra contribute the stellar component(s) of the SED model, while the dust emission is modeled as a blackbody. By varying the temperatures of the stars and dust, as well as their effective radii, the composite curve was obtained using a $\chi^{2}$ minimization fit to the available photometric data of both the bright and faint states. Given the multi-parameter fit, we have improved upon the analysis of Plavchan et al. (2008a) to include an AMOEBA simplex code parameter optimization (Nelder \& Mead 1965). Figure 4 shows the best model SED fits to each of the bright and faint state SEDs, and the best fit parameters are given in Table 2. Simplex codes are generally sensitive to the initial guesses. Thus, our fit likely represents only one of multiple degenerate models that can adequately describe the SED in both the bright and faint states. However, we have chosen to fix some of the parameters to enforce ensure that these parameters remain consistent between the bright and faint states, and given the limited degrees of freedom.

\section{Discussion}

From the NACO images in Fig. 1, we confirm the presence of at least two YSOs in the YLW 16A system. The $L$ band NACO image shows two sources of approximately equal brightness, whose projected separation is around $0.3^{\prime \prime}$, corresponding to a projected separation of $\sim 40 \mathrm{AU}$ at a distance of $135 \mathrm{pc}$. The $K_{\mathrm{s}}$ band NACO image shows a more complex nebulosity surrounding the visual binary, indicating at least one component of the binary is deeply embedded in a protostellar envelope. Both NACO images were obtained in the faint state ( $L$ band phase $=0.4656$, $K_{\mathrm{s}}$ band phase $\left.=0.6920\right)$. These images do not constrain which of the visual components is the source of the observed photometric variability. However, if the stellar components are of equal spectral type and radii, the fainter $K$-band component is a likely candidate given that the system was in a faint state at the time.
Table 2. SED model parameters.

\begin{tabular}{ll}
\hline \hline $\begin{array}{l}\text { Parameter } \\
\text { fixed }\end{array}$ & Value \\
\hline $\begin{array}{l}\text { Distance } \\
\text { Short- } \lambda \text { extinction power law } \alpha\end{array}$ & $135 \mathrm{pc}$ \\
Long- $\lambda$ extinction power law $\alpha$ & $-2.0^{a}$ \\
\hline \multicolumn{2}{l}{ Varying in faint state fit, fixed in bright state fit to faint fit } \\
\hline$\lambda$ Extinction transition & $4.0 \mu \mathrm{m}^{a}$ \\
Cold dust $T, L$ & $91.3 \mathrm{~K}, 1.92 L_{\odot}{ }^{b}$ \\
Cold dust $A_{J}$ & $4.2 \mathrm{mag} b$ \\
\hline \multicolumn{2}{c}{ Varying, best fit, faint state } \\
\hline Composite $T_{*}, R_{*}, L_{*}$ & $3514 \mathrm{~K}, 4.27 R_{\odot}, 2.49 L_{\odot}$ \\
$A_{J}$ stellar extinction & $9.86 \mathrm{mag}$ \\
Hot dust $T, L$ & $562 \mathrm{~K}, 1.10 L_{\odot}$ \\
$A_{J}$ hot dust & $0 \mathrm{mag}{ }^{c}$ \\
\hline \multicolumn{2}{c}{ Varying, best fit, bright state } \\
\hline Composite $T_{*}, R_{*}, L_{*}$ & $3525 \mathrm{~K}, 4.66 R_{\odot}, 3.01 L_{\odot}$ \\
$A_{J}$ stellar extinction & $10.1 \mathrm{mag}$ \\
Hot dust $T, L$ & $579 \mathrm{~K}, 1.63 L_{\odot}$ \\
$A_{J}$ hot dust & $0.57 \mathrm{mag}$ \\
\hline
\end{tabular}

Notes. ${ }^{(a)}$ Extinction wavelength dependence adopted from Becklin et al. (1978); Mathis (1990). A transition-wavelength initial guess of $3.5 \mu \mathrm{m}$ was used in the fit, but was allowed to float freely in the fit to the faint state photometry. The fit for the bright state photometry fixed the transition-wavelength to that of the best fit in the faint state. (b) When we allow the cold dust temperature and luminosity to vary in our SED fit to the bright state photometry, the effect on the best fit parameters is marginal: $<1 \mathrm{~K},<1 \%$ luminosity, $\sim 0.1$ mag $A_{J}$ extinction difference. Thus, we fix these values in the bright state fit to reduce the degrees of freedom. The $A_{J}$ value for the cold dust is larger than the best fit hot dust extinction, which seems counterintuitive. This may relate to non-blackbody thermal dust grain emission, or the cold and hot dust being associated with different components of the visual binary. ${ }^{(c)}$ Negative extinction magnitudes were not allowed in the fit.

Time-resolved adaptive optics monitoring is necessary to confirm which component contributes to the photometric variability.

Our SED model fit confirms the Class I nature of YLW 16A. A fit with two dust blackbody components and no stellar components yields an unphysical extinction power law, and thus the SED model necessitates at least one stellar component. The large extinction of the stellar components and potential differences in infrared excess do not motivate/yield any useful constraints on multiple component stellar radii nor temperatures, even though the NACO images indicate at least two components. For example, a higher stellar temperature is partially degenerate with a larger stellar extinction. Rather, we derive a single composite set of stellar parameters from the fit of $T_{*} \sim 3500 \mathrm{~K}$ and effective stellar radius of $\sim 4.66 R_{\odot}$. If the system consists of three stars with equal radii and temperatures in the bright state, the corresponding stellar radii of each of the three components would be $\sim 2.7 R_{\odot}$. If the system consists of two stars with equal radii in the faint state - e.g., a third star is completely extincted/eclipsed by a primordial disk - the corresponding stellar radii of each of the two components would be $\sim 3.0 R_{\odot}$. These radii are plausible for Class I YSOs. Our value of $A_{J} \sim 10$ yields a visual extinction much larger than that derived in Evans et al. (2009), despite providing an initial guess for the stellar extinction in our model SED fit of $A_{J}=2$.

The 92.6 day periodic photometric variability cannot be associated with the Keplerian orbit of the projected $\sim 40$ AU visual 


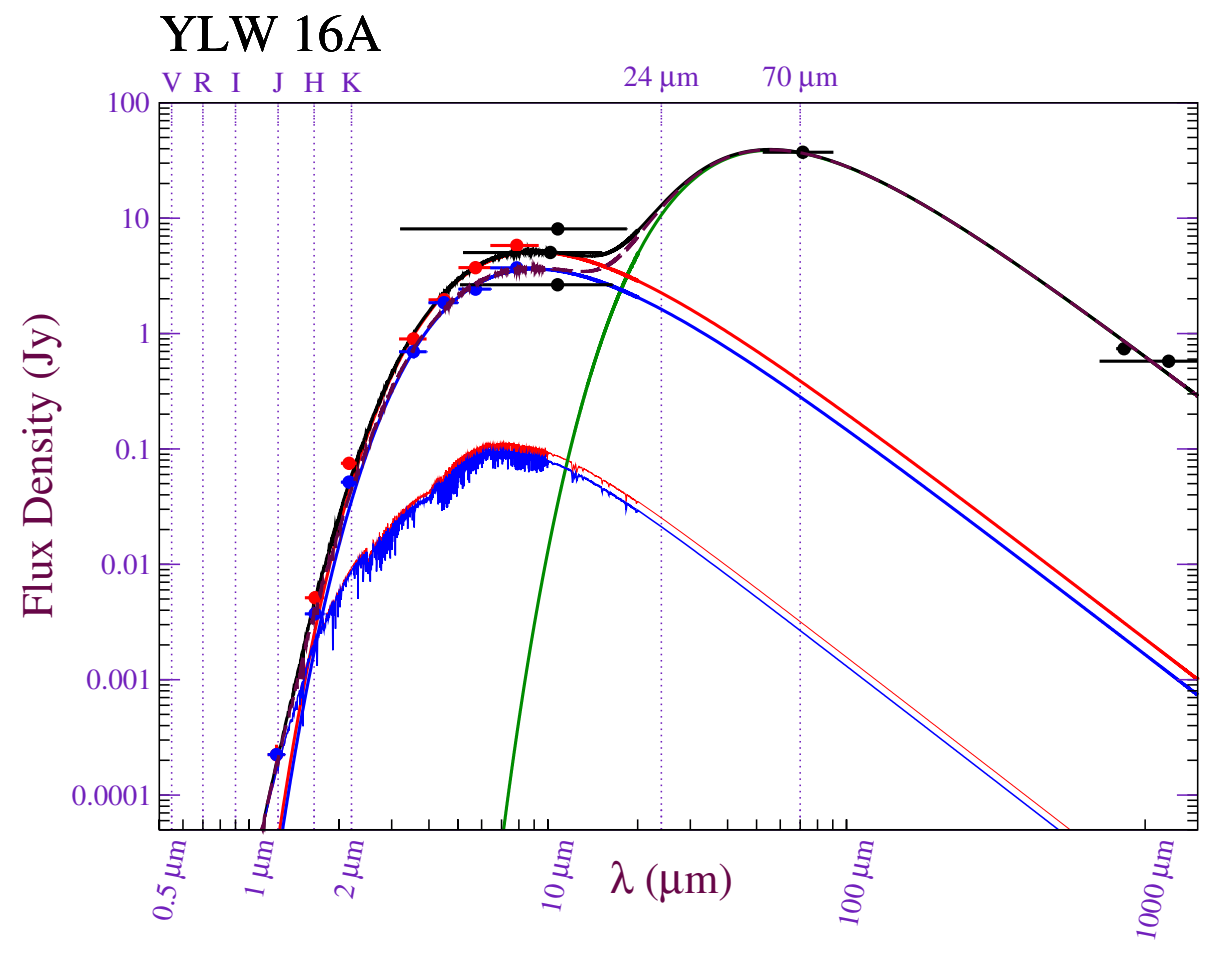

Fig. 4. The model SED fit to observed YLW 16A photometry. Red circles correspond to JHK and Spitzer IRAC photometry during the bright state; blue circles correspond to the faint state. The summed model SED fit (black curve: bright state; maroon dashed curve: faint state) has contributions from a composite star (lower synthetic SEDs peaking at $\sim 0.1 \mathrm{Jy}$; red: bright state; blue: faint state), hot dust (upper left blackbody curves peaking at a few Jy, red: bright state; blue: faint state), and cold dust (green blackbody curve). Ground-based historical $10.8 \mu \mathrm{m}$ photometry, Spitzer MIPS 70 micron photometry and sub$\mathrm{mm}$ photometry is shown as black circles (YLW 16A is saturated at $24 \mu \mathrm{m}$ in the Spitzer c2d survey data). companion. Invoking the discussion in Plavchan et al. (2008a), the periodic photometric variability of YLW 16A is not readily attributable to starspots, chaotic disk extinction from accretion, nor other stellar activity induced variations that tend to operate on time-scales of less than a week. Instead, we postulate that the long-term periodic photometric variability of YLW 16A indicates the presence of a tertiary companion of unknown mass within a few AU of one of the visual components and with an orbital period of 92.6 days. If the tertiary companion is approximately the same mass/brightness of the other two companions, and it is periodically eclipsed by a circum-binary disk, this scenario could explain both the periodic variability as well as the $\sim 1 / 3$ reduction in flux between bright and faint states. The lack of a detection of binarity in Covey et al. (2006); Doppmann et al. (2005) indicates that this tertiary companion may instead be at a much smaller observed luminosity. The SED model indicates that the luminosity of the hot dust component must also change substantially to explain the observed variability at IRAC mid-IR wavelengths. In other words, at $K_{\mathrm{s}}$ and IRAC bands, we are seeing possible periodic eclipses (shadowing) of some of the hot dust material in the system associated with this tertiary companion, rather than the proto-star photosphere itself. This in turn implies a strong star-disk dynamical interaction.

The literature photometry for YLW 16A provides an indication of the stability of the system, supporting a Keplerian origin to produce the observed periodic variations. $K_{\mathrm{s}}$ band photometry from Simon et al. (1987) and Wilking et al. (1989), as given in Table 1, indicates that the bright state magnitude of $\sim 9.8$ may have been consistent for over twenty years. However, the faint state value of $\sim 8.8 \mathrm{mag}$ does not agree with our observations. This could be due to long-term evolution in primordial disk structure. Follow-up observations with the Spitzer Space Telescope and the YSOVAR program will further constrain the long-term stability of the observed periodic variability (Morales-Calderon et al. 2011).

Finally, the discovery of a system similar to WL 4 indicates that such systems may be common, and more may be uncovered with long-term photometric NIR and mid-IR intense photometric monitoring. Both WL 4 and YLW 16A possess visual companions at wide separations. This implies that a wide companion offers a plausible, if not required, mechanism to explain how a circum-binary disk could be warped with respect to the orbit of a hypothesized inner binary, to produce the observed periodic disk extinction. These two systems join KH-15D in NGC 2264 and CHS 7797 in the Orion star-forming region (Herbst et al. 2010; Rodriguez-Ledesma et al. 2013, 2012).

\section{Conclusion and future work}

We identify 92.6 day periodic photometric variability for the YSO YLW 16A. We confirm the system is also a visual binary with a projected separation of $\sim 40 \mathrm{AU}$. We infer a possible triple system for YLW 16A, similar to the model proposed for WL 4 by Plavchan et al. (2008a), indicating such systems may be common.

The nature of the companion producing the observed periodic photometric variations is unknown. High S/N NIR spectroscopic monitoring of the 2.3 micron $\mathrm{CO}$ feature for radial velocity variations over three months, especially during the bright state, may confirm the presence of a tertiary companion responsible for producing the observed photometric variations. Synoptic 3.6 and $4.5 \mu \mathrm{m}$ observations have been obtained for both WL 4 and YLW 16A as part of the YSOVAR Spitzer program (Morales-Calderon et al. 2011). Preliminary analysis indicates that the sources retain the photometric variability expected if the variability is driven by a Keplerian companion. The analysis of this data will be reported in a future publication. After the paper was accepted for publication, it was brought to the authors' attention that an independent analysis of the same archival images from NACO was published in Duchene et al. (2006).

Acknowledgements. The authors would like to thank the anonymous referee for the manuscripts review. We thank Karl Stapelfeldt, John Stauffer, Lynne Hillebrand and Andreas Seifahrt for their critical comments and discussion. This 
research has made use of the NASA Exoplanet Archive, which is operated by the California Institute of Technology, under contract with the National Aeronautics and Space Adminstration under the Exoplanet Exploration Program. This work is based (in part) on observations made with the Spitzer Space Telescope, which is operated by the Jet Propulsion Laboratory, California Institute of Technology under a contract with NASA. Support for this work was provided by NASA through an award issued by JPL/Caltech. This research has made use of the NASA/IPAC Infrared Science Archive, which is operated by the Jet Propulsion Laboratory, California Institute of Technology, under contract with the National Aeronautics and Space Administration.

\section{References}

Adams, F. C., Lada, C. J., \& Shu, F. H. 1987, ApJ, 312, 788

Allen, L. E., Myers, P. C., Di Francesco, J., et al. 2002, ApJ, 566, 993

Barsony, M., Ressler, M. E., \& Marsh, K. A. 2005, ApJ, 630, 381

Beckford, A. F., Lucas, P. W., Chrysostomou, A. C., \& Gledhill, T. M. 2008, MNRAS, 384, 908

Becklin, E., Matthews, K., Neugebauer, G., \& Willner, S. 1978, ApJ, 220, 831

Bertone, E., Buzzoni, A., Chavez, M., \& Rodriguez-Merino, L. H. 2004, AJ, 128,829

Bontemps, S., Andre, P., Kaas, A., et al. 2001, A\&A, 372, 173

Covey, K., Green, T., Doppman, G., \& Lada, C. 2006, AJ, 131, 512

Doppmann, G. W., Greene, T. P., Covey, K. R., \& Lada, C. J. 2005, AJ, 130, 1145

Doyle, L., Carter, J., Fabrycky, D., et al. 2011, Science, 333, 1602

Duchene, G., Beust, H., Adjali, F., et al. 2006, A\&A, 457, 9

Evans, N. J. II, Allen, L., Blake, G., et al. 2003, PASP, 115, 965

Evans, N. J. II, Dunham, M., Jorgensen, J., et al. 2009, ApJS, 181, 321

Faesi, C., Covey, K., Gutermuth, R., et al. 2012, PASP, 124, 1137

Flaherty, K. M., \& Muzerolle, J. 2010, ApJ, 719, 1733

Flaherty, K. M., Muzerolle, J., Rieke, G., et al. 2012, ApJ, 748, 71

Greene, T. P., \& Lada, C. J. 2000, AJ, 120, 430

Girart, J. M., Curiel, S., Rodriguez, L. F., et al. 2004, AJ, 127, 2969

Grosso, N. 2001, A\&A, 370, L22
Hauschidlt, P., Allard, F., \& Baron, E. 1999, ApJ, 512, 377

Herbst, W., LeDuc, K., Hamilton, C., et al. 2010, AJ, 140, 2025

Herczeg, G., Brown, J., van Dishoeck, E., \& Pontoppidan, K. 2011, A\&A, 533, 112

Ida, S., \& Lin, D. N. C. 2010, ApJ, 719, 810

Imanishi, K., Koyama, K., \& Tsuboi, Y. 2001, ApJ, 557, 747

Johnstone, D., Di Francesco, J., \& Kirk, H. 2004, ApJ, 611, L45

Jrgensen, J. K., Johnstone, D., Kirk, H., et al. 2008, ApJ, 683, 822

Joy, A. 1945, ApJ 102, 168

Kovacs, G., Zucker, S., \& Mazeh, T. 2002, A\&A, 391, 369

Lenzen, R., Hartung, M., Brandner, W., et al. 2003, SPIE, 4841, 944

Leous, J. A., Feigelson, E. D., Andre, P., \& Montmerle, T. 1991, ApJ, 379, 683

Lin, D. N. C., \& Papaloizou, J, 1980, MNRAS, 191, 37

Lucas, P. W., \& Roche, P. F. 1998, MNRAS, 299, 699

Luhman, K. L., \& Rieke, G. H. 1999, ApJ, 525, 440

Mamajek, E. E. 2008, Astron. Nachr., 329, 10

Marsh, K. A.., Plavchan, P., Kirkpatrick, J., et al. 2010, ApJ, 719, 550

Mathis, J. S. 1990, ARA\&A, 28, 37

Morales-Calderon, M., Stauffer, J., Hillenbrand, L., et al. 2011, ApJ, 733, 50

Nelder, J. A., \& Mead., R. 1965, Comput. J., 7, 308

Padgett, D. L., Rebull, L., Stapelfeldt, K., et al. 2008, ApJ, 672, 1013

Parks, J. R., Plavchan, P., White, R., \& Gee, A. H. 2013, ApJS, submitted

Plavchan, P., Gee, A. H., Staplefeldt, K., \& Becker, A. 2008a, ApJ, 684, L37

Plavchan, P., Jura, M., Kirkpatrick, J. D., Curti, R. M., \& Gallagher, S. C. 2008b, ApJS, 175, 191

Rebull, L. 2001, AJ, 121, 1676

Rodriguez-Ledesma, M. V., Mundt, R., Ibrahimov, M., et al. 2012, A\&A, 544, A112

Rodriguez-Ledesma, M. V., Mundt, R., Pintado, O., et al. 2013, A\&A, 551, A44

Rousset, G., Lacombe, F., Puget, P., et al. 2003, SPIE, 4839, 140

Scargle, J. D. 1982, ApJ, 263, 835

Simon, M., Howell, R. R., Longmore, A. J., et al. 1987, ApJ, 320, 344

Skrutskie, M. F., Cutri, R., Stiening, R., et al. 2006, AJ, 131, 1163

Stanke, T., Smith, M. D., Gredel, R., \& Khanzadyan, T. 2006, A\&A, 447, 609

Wilking, B. A., Lada, C. J., \& Young, E. T. ApJ, 340, 823

Xiao, H. Y., Covey, K., Rebull, L., et al. 2012, ApJS, 202, 7 